\title{
Embedded VANETs Architecture
}

\author{
Madiha Sarwar \\ Department of Computer \\ Science \\ The University of Lahore \\ Sargodha, Pakistan
}

\author{
Komal Kamran \\ Department of Computer \\ Science \\ The University of Lahore \\ Sargodha, Pakistan
}

\author{
Ahmed Farid \\ Department of Computer \\ Science \\ The University of Sargodha \\ Sargodha, Pakistan
}

\begin{abstract}
Vehicular ad hoc network (VANETs) is considered one of the ways of providing Intelligent Transportation Services (ITS) to Vehicular Nodes (VN). In order to help out the driver, the main issue is to allocate the exact position of the $\mathrm{VN}$ through some Base station. Most of the navigational decision support can be provided by Base stations even in low visionary situations. The goal is to get the exact position of $\mathrm{VN}$ and for this embedded architecture is proposed along with OFDM. In this study two different VANETs architectures are compared and an existing architecture modified.
\end{abstract}

\section{Keywords}

VANETs (Vehicular Ad Hoc Networks), MIMO OFDM (multiple input multiple output-orthogonal frequency multiplexing), ITS (intelligent transportation system), WSN (wireless sensor networks), V2V (Vehicle-to-Vehicle communication), V2R (Vehicle-to-Roadside Communication)

\section{INTRODUCTION}

A new kind of embedded system is in vehicles to communicate with each other and possibly with a roadside infrastructure and make a new kind of ad hoc network (VANETs), to provide a long list of applications varying from transit safety to driver assistance and Internet access. On these networks, information about the real-time position of nodes is an important issue and discussed by most architectures. For this purpose commonly GPS receivers can be installed easily in vehicles, a number of which already comes with this technology. But as VANETs advances more, GPS starts to show some undesired problems such as not always being available or not efficient to handle some applications [1-3].

Embedded vehicles are equipped with communication equipment that allows them to exchange messages with each other in Vehicle-to-Vehicle communication (V2V) and also to exchange messages with a roadside network infrastructure Vehicle-to-Roadside Communication (V2R) having processor, sensor, GSM, generators and memory modules (ROMS). A number of applications are envisioned for these networks, some of which are already possible in some recently designed vehicles:

- Vehicle collision warning

- $\quad$ Security distance warning

- Driver assistance

- Cooperative driving

- Cooperative cruise control

- Dissemination of road information

- Internet access

\section{- Map location}

The point which is the most important in embedded VANETs is to figure out the vehicular node's location with accuracy. There are two main reasons to focus on this point; one is to get the proper track of vehicle along with its neighboring vehicles, so that we must be able to inform vehicles with the help of alarm in dangerous situations. The other is, to exchange data with the security. Data security in VANET is getting importance because it has to deal with two issues, because when two vehicle transfer data to each other then there is a probability that the data of one vehicle can go to the vehicle which is not intended, this can cause error in ad-hoc control navigation system.

For this MIMO OFDM (multiple input multiple output orthogonal frequency multi-plexing) used. It has different antennas, which transfer signals of different frequencies. And on the other side the same different antennas receive their concern signals and so that they cannot interact with each other.

The main goal of embedded vehicle is to provide safety to the passengers. For this each vehicle is equipped with electronic devices that can communicate with other vehicles as well as infrastructure. Emergency, warning and alert messages guide the drivers to choose the best path along the way. Passengers can also get internet access, automatic payment of parking lots and tolls are another example of the possibilities in VANET.

When discuss about VANET, it is important to discuss about wireless sensor networks. The main advantages of using sensor are that they provide better connectivity with the base station and tell exact locations of the accidents etc.

Organization of this paper is as follows: In Section 2 Literature review is presented, section 3 provides comparison between two architectures and section 4 presents proposed scheme. Finally, this paper is concluded in section 5.

\section{LITERATURE REVIEW}

In VANET, each vehicle has to remain aware of other vehicle's position or speed and its surrounding etc. For this purpose, every car periodically transmits a short status message (beacons). We are going to purpose a beaconing strategy for car to car communication in VANET. We have assumed that there are a fixed number of vehicles on the road. The vehicle which wants to transmit beacon, we call it active and others are called inactive [4].

Communication and information collection between two VANETS is important in intelligent transportation system (ITS) [7]. Mostly ITSs detects only vehicles in fixed positions and for this purpose they use communication and power cables that increases the cost of construction and maintenance. 
Comparatively wireless sensor networks (WSN) are low cost wireless distribution and flexible as there is no restriction of cable. Firebase MAC protocol [9] can save energy, adapts itself according to traffic load. We want minimal RSU support as the vehicle enters or exit rapidly, so we want a protocol which requires minimal RSU support and efficient handoff from one RSU to another. We require a directional antenna as the vehicles are moving on the road. By using shared wireless communication is through broadcast. RSU periodically broadcasts its location and the vehicles that are in its range receive its broadcast, the vehicle calculates its position and sends the association request to the RSU. RSU receives the request and maintain the table that which of the channels, assign to which node for which amount of time or transmission is unidirectional and bidirectional. RSU also maintains a matrix for recording the free or allocated channels. RSU solves the hidden terminal problem by refusing a channel who wants to communicate with its neighbor and the neighbor is busy in another communication. Traffic is a device that can be embedded in the vehicles so that drivers can see the real time road traffic view will help drivers to see far beyond than they can see physically.

The vehicles that are equipped with the traffic view can disseminate traffic information using a short range wireless communication.

\subsection{V2V Wireless Communication}

In this V2V wireless communication the 802.11 [11] DCF modes have been modified in a way so that in communication there is a low latency in the propagation of packets. In original DCF the every failure occurs, it doubles the contention window size and successful channel access decrease the value of BEB. Here it is enhanced in a way.

\subsection{VADD (Vehicle Assisted Data Delivery in VANET)}

Based on the idea of carry and forward. Its aim is to select a forwarding path with the smallest packet delivery delay [1]. So, VADD follows the basic principles.

i. It uses wireless transmission mediums, as much as possible.

ii. If the certain roads are used to carry data, then it will choose a road with higher continuously be executed. Throughout the packet-forwarding process, because VANET has an unpredictable nature [11].

Location First-Probe (L-VADD) and ("Direction - First Probe") [1]. It gives preferred forwarding direction of a packet.

\subsection{Direction First Probe}

Gives closest contact to VADDI that detection as the next hop. But problem is that when L-VADD works, then routing loop can occur. That can affect the performance of protocols and gives lower packet - delivery ratio [2]. It can be controlled by a loop detection mechanism but it will increase forwarding delay and of D-LADD is free from routing loop, but some time, it may suffer from a long packet forwarding distance and long packet delivery delay. To overcome these problems, H-VADD is composed that inherits the ability of shortest forwarding path in L-VADD when there is no routing loops and uses D-VADD to control the loop problem of LVADD [2].

\section{Feasibility of a 802.11 VANET car accident alert system}

In VANET, presently the process of reporting car accidents is extremely slow [3].

The accident must be first reported physically, and then it should be broadcasted in the network. Moreover, drivers must have a choice to take alternative routes if possible [8]. For this, the car accident alert system must have two threads, one thread will detect the car is in accident or not. If yes, the warning messages must be broadcasted in that zone. The second thread will listen these messages and that the CW size remain fix for emergency warning messages only, if channel access failure it size will not double therefore there will be so less delay factor in the emergency warning messages propagation further it has no retry limitations.

Warn the drivers about the danger, and if driver wants to change the route, second thread will provide him related information [3].

Now we will describe the design and working of car accident alert system, first system will collect raw acceleration data through an accelerometer, this data will then go to the controller where it will be detected that accident has been occurred or not. If accident is detected, the controller requests the GPS in order to obtain the car's current location as well as maximum acceleration, through wireless transceiver. In the meantime, the controller also listens for these messages through a wireless transceiver [9]. If message receives, the controller will obtain the relative distance of the accident through GPS. At the end, the controller provides audio alert through speakers which will alert the drivers about the danger [3].

\subsection{Real Time Car to Car Communication Protocol for intersecting Roads}

In VANET, real time communication is needed for safety related tasks such as emergency warning messages, etc. For real time communication in VANETs, time bounded medium access is very necessary [4].

In this protocol, we provide a bounded delay to access the medium for all vehicles by assigning a unique time slot to each vehicle, to broadcast the messages .This scheme works in both, highways and urban scenarios. Our main goal is to avoid the effect of interference due to communication of vehicles travelling on adjacent roads that are within the communication range of each other, such as there come an intersection [4]. We are proposing a communication framework for intersecting roads that is based on Space-time Division Multiple Access (SDMA) that assures each vehicle can access the medium within the required time bound.

In existing SDMA scheme, there is a problem, the same time slot is reused by the vehicles on two different locations that are separated by a distance at least equal to twice the sensing range [4]. This condition is necessary in SDMA, in order to prevent message losses, but this condition is not guaranteed when more roads lie near an intersection. This condition results in packet collision, because two vehicles in different cells assign a same slot and they broadcast message at the same time [12].

Here are some points that can be the solution of this problem:

- Each cell must obtain an equal share of bandwidth or time slot. 
- $\quad$ Time slots must be equally distributed between all roads.

- No message should be lost due to packet collision.

- Two cells on the same road can be assigned the same time slot only if these cells have separate distance of at least twice of sensing range [4].

When beacon will be lost, active vehicle will send new beacon that replaces the old one and old one will be assumed at lost [2].

\subsection{Traffic View: A driver assistant device for traffic monitoring based on car to car communication}

Traffic is a device that can be embedded in the vehicles so that drivers can see the real time road traffic view will help drivers to see far beyond than they can see physically [3].

The vehicles that are equipped with the traffic view, can disseminate traffic information using a short range wireless communication,

Traffic view device is composed of three components, an embedded computer with display; GPS receiver and a shortrange wireless network interface [3]

The GPS receiver provides the location, speed, time and direction of vehicles etc.

Each that is equipped traffic view can gather information about other vehicles in the network [3].

Drivers of each vehicle can exchange information with each other, using the limited bandwidth [3].

We have focused on three issues i.e. estimating VN position using GPS, calculating relative distances among the VN's using some sensor network techniques and communication among the VN's using OFDM. The brief descriptions of issues are discussed in this section.

Joseph et all. presented different terminologies for GPS using GSM are discussed along with their coding schemes and disciplines. In radio service that uses circuit switched which is an efficient thing, especially in-burst transmission because of recourse reservation for error correction purpose GPRS defines four encoding schemes (cs1, cs2, cs3, cs4) in RLC/MAC for data transfer and security purposes. Details about the schemes can be found in.

Yan Zhang and Boon-Hee Soong presented the scheme which reallocates the channel for GPRS which significantly decrease the voice call blocking probability and GPRS packet transmission time slight channel and negligible expense on GPRS packet blocking probability. Basically RASreallocation scheme is discussed in this paper which utilizes the idle channels for reallocation. Reallocation of the channels mainly depends upon the number of arrivals for voice calls and amount of GPRS data. So channels are reallocated and they help to reduce the blocking probability and reduce GPRS packet transmission time, which also helps in reducing the expense of connectivity.

Various MIMO-OFDM key techniques are introduced. Some of our considerations in MIMO-OFDM system focus on frame structure, CE, and comparisons of STC and spatial multiplexing. The proposed frame structure with scattered pilots is especially suited for high-data-rate transmission at high mobility. Based on comparisons of STC and spatial multiplexing, qualitative criteria in terms of data rate, application environment, and antenna configuration are proposed to choose STC or spatial multiplexing. Link level simulation results for the designed MIMO-OFDM spatial multiplexing system presents encouraging performance. The high bandwidth efficiency obtained which shows that MIMOOFDM is a potential candidate for future broadband wireless Access.

Yan Wang, Moshe Zukerman and Richard J. Harris presented new mobile service that enables fast access and two way communication between two or than two nodes. GSM use the TDMA structure with 8 time slot to support voice and data transmission we consider typical GPRS/GPS network using partial sharing scheme. The working of the scheme is as fallow:

Jie Du and Matthew J. Barth methodology is based on using fixed infrastructure in which base station receives the satellite data via global positioning system (GPS) and sends corrections (after calculating the offset) to the car or rover through wireless communication. After having the position information from the $\mathrm{VN}$ then it calculates the lane position using a map which is installed in the base station. It also logs the position and lane parameter for each rover into a database for a subsequent analysis. Through experiments, it is shown that it gives $2 \mathrm{~m}$ level positioning accuracy and sends its positions speed and vehicle status to the base station on a periodic basis. For accuracy GPS in cooperated with DGPS.

\subsection{Design and Architecture}

We have used a hybrid approach in design because our main emphasize on finding the exact location of the VN. For this purpose, we have merged both the techniques that have been defined. The intention is to have a decision support system at the Base station. The Base station will decide which parameters are more important while servicing the requests of VN's. For example, if weather conditions are not good, then there will be some way to have additional information about the VN locations. Under the normal conditions GPS and DGPS will provide the enough information to calculate the VN position, but if base station requires additional information then for this purpose Y-CAM will be in cooperating with it that will help in calculating the exact position. Furthermore, after having the exact position coordinates the communication process in carried out through GPRS. If the VN's are interested to have relative position coordinates, then these requested parameters will get the response through GPRS. Lastly, if the VN intended to have communication sessions with some other $\mathrm{VN}$ then by using MIMO-OFDM this can also be possible. The important information about using OFDM will also be exchanged through GPRS. In this case, each vehicle should be connected to MIMO OFDM antenna which receives the signals from other vehicles.

The traffic view device is composed of three components, an embedded computer with display; GPS receiver and a shortrange wireless network interface

The GPS receiver provides the location, speed, time and direction of vehicles etc.

Each that is equipped traffic view can gather information about other vehicles in the network. 
Drivers of each vehicle can exchange information with each other, using the limited bandwidth.

We have focused on three issues, i.e. estimating VN position using GPS, calculating relative distances among the VN's using some sensor network techniques and communication among the VN's using OFDM. The brief descriptions of issues are discussed in section $[5,6]$.

\section{COMPARISON OF VANET ARCHITECTURES}

In Rahat's architecture possible communication is vehicle to road side unit and vehicle to vehicle by using dedicated short range communication technology using on-board screen. and this system will inform about any kind of accident and mishap to RSU and through RSU to other vehicles.

Ahmed's Architecture is using a global navigation satellite system (GPS) that will determine the position of vehicles, show derived information like direction and speed of vehicles calculated from position changes. Using GSM kit the node will act as a part of cellular network so that transmission of voice and text messages is easily available, it makes it very convenient for international travelers to connect to emergency services without having any knowledge of local emergency numbers. This architecture uses a memory module to keep a record of data transmitted through GSM and GPS as well and presence of heat and smoke sensor make this architecture more reliable.

\subsection{Rahat Ali Khan's Architecture}

The main concern of this Architecture is to manage and control traffic flow like safely driving and accident avoidance. In this architecture vehicle to vehicle (V2V) and vehicle to infrastructure (V2I) communication is possible for the concerns as explained above. The road side unit (RSU) act as a base station to transmit and receive messages which are generated by any kind of incident occur. This communication will be possible with the help of on board sensors.

Warning control sensors will generate warnings for traffic rules violation and about road conditions. Roadside unit (RSU) will locate near the place this unit will receive messages and generates automatic messages to other vehicles so they might not come towards the place. Lane detection will tell in which lane accident has occurred so, it will give the warning to avoid lane changing again and again [5].

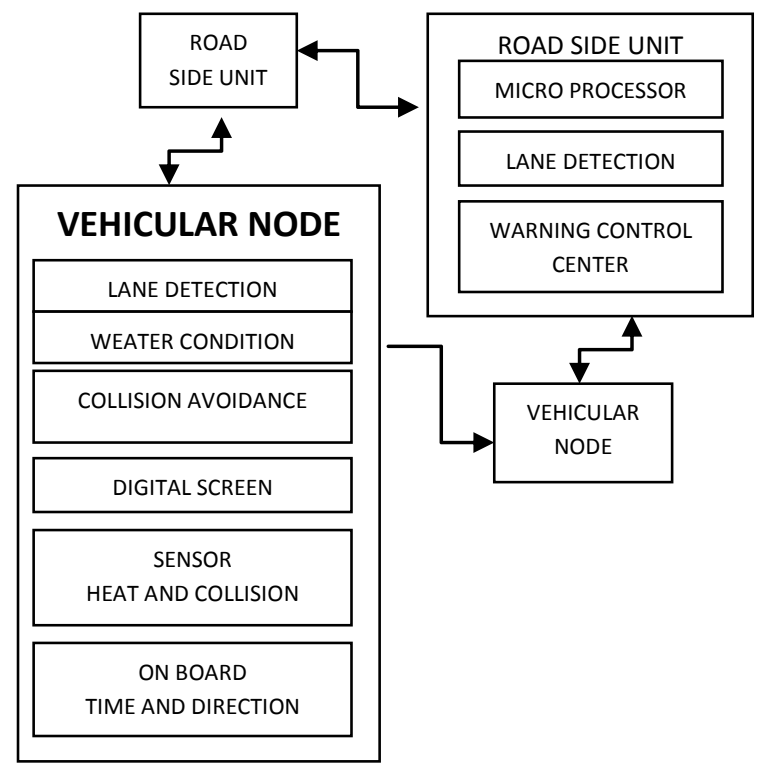

Fig1: Architecture diagram of intelligent transportation system [5]

\subsection{Ahmed Farid's Architecture}

This architecture is designed to avoid fire emergency with a wireless sensor network design for highways, so that it can easily adapt traffic and weather conditions. It focuses on high packet delivery of messages with low latency.

Micro controller provides connectivity to all other hardware components; EP ROM is used as a memory module to save GPS and sensor data. GSM kit is used to provide support for cellular networks that is further connected with GPS system.

GPS tracker will track the car ids in its zone and cell numbers of vehicles will be recorded in the microcontroller's memory these numbers will be helpful in sending broadcast and unicast messages through GSM [6].

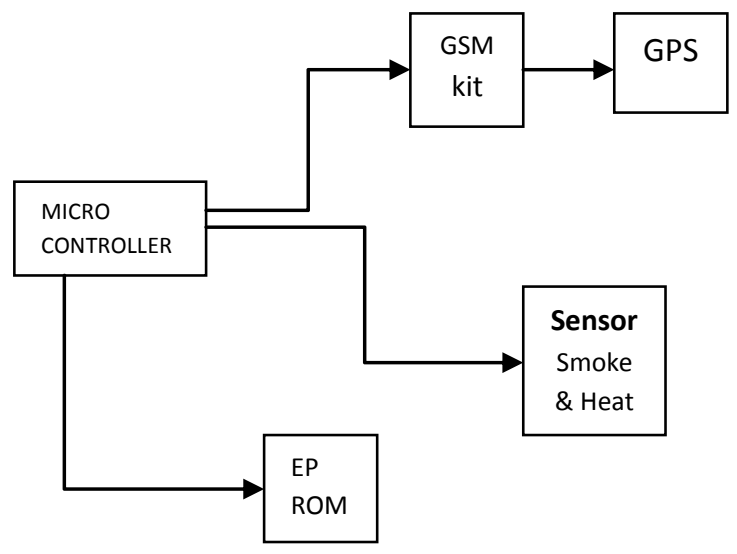

Fig2: Architecture diagram of Wireless sensor embedded system [6]

\section{PROPOSED ARCHITECTURE}

We have used a hybrid approach in design because our main emphasize on finding the exact location of the VN. For this purpose, we have merged both the techniques that are GPS and DGPS. The intention is to have a decision support system at the Base station. The Base station will decide which 
parameters are more important while servicing the requests of VN's.

For example, if weather conditions are not good, then there will be some way to have additional information about the VN locations. Under the normal conditions GPS and DGPS will provide the enough information to calculate the $\mathrm{VN}$ position, but if base station requires additional information then for this purpose Y-CAM will be in cooperated with it that will help in calculating the exact position.

Furthermore, after having the exact position coordinates the communication process in carried out through GPRS. If the VN's are interested to have relative position coordinates, then these requested parameters will get the response through GPRS. Lastly, if the VN intended to have communication sessions with some other VN then by using MIMO-OFDM this can also be possible.

The important information about using OFDM will also be exchanged through GPRS. In this case, each vehicle must be connected to MIMO OFDM antenna which receives the signals from other vehicles.

GPS exchanges data with DGPS (differential GPS) which provides more accuracy and with the help of MIMO OFDM this architecture is providing multiple frequencies for the sake of secure transmission of data between cars and central station. It provides the internet facility using wireless cloud as well. Our proposed architecture is scalable for more sensors like smoke, heat, parking and accident alert sensors.

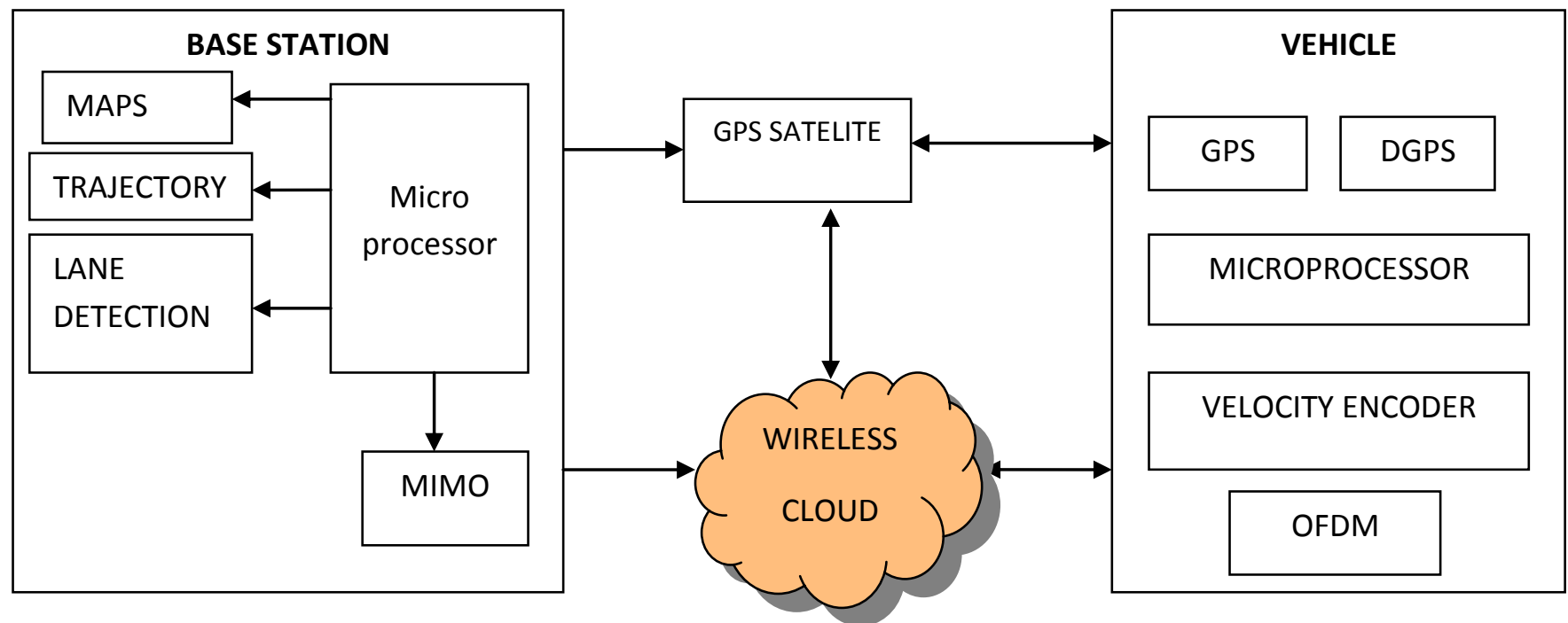

Fig3: Architecture diagram of proposed system

\section{CONCLUSION}

The proposed architecture focuses on retrieving the exact position of Vehicular node through the inclusion of DGPS, having an accurate position of $\mathrm{VN}$ makes this architecture better than the other two and also enhances the traffic intelligence moreover velocity is also calculated at the $\mathrm{VN}$, so this decreases the computational delays at Base station. The inclusion of MIMO in purposed system provides the ease of $\mathrm{VN}-\mathrm{VN}$ communication.

\section{REFERENCES}

[1] S. Biswas, R. Tatchikou, F. Dion, Vehicle-to-vehicle wireless communication protocols for enhancing highway traffic safety, IEEE Communications Magazine 44 (1) (2006) 74-82.

[2] J. Blum, A. Eskandarian, L. Hoffman, Challenges of inter-vehicle ad hoc networks, IEEE Transactions on Intelligent Transportation Systems, pp 347-351,2004.

[3] W. Kiess, J. Rybicki, M. Mauve, On the nature of intervehicle communication, in: WMAN 2007: Proceedings of the 4th Workshop on Mobile Ad-Hoc Networks, 2007, pp. 493-502.Tavel, P. 2007 Modeling and Simulation Design. AK Peters, Ltd.
[4] Alexey Vinel, Dirk Staehle and Andrey Turlikov "Study of beaconing for car-to-car communication in vehicular ad-hoc networks, 2009 IEEE

[5] Rahat Ali Khan, Shakeel Ahmed Shah, Muhammad Abdul Aleem, Zulfiqar Ali Bhutto, Asad Ali Shaikh, Muhmaad Aslam Kumbhar, "Wireless Sensor Networks: A Solution for Smart Transportation", Journal of Emerging Trends in Computing and Information Sciences, VOL. 3, NO. 4, April 2012.

[6] Farid, Ahmed; Mushtaq, Muhammad Azhar; Kaleem, Muhammad; Nawaz, Sana. "Preparation of FBMH and EFBWSN: A Firebase MAC Protocol and Enhanced WSN Design for Highways"

[7] Hartenstein, Hannes, and Kenneth P. Laberteaux. "A tutorial survey on vehicular ad hoc networks." Communications Magazine, IEEE 46, no. 6 (2008): 164171.

[8] Sichitiu, Mihail L., and Maria Kihl. "Inter-vehicle communication systems: a survey." Communications Surveys \& Tutorials, IEEE 10, no. 2 (2008): 88-105.

[9] Karagiannis, Georgios, et al. "Vehicular networking: A survey and tutorial on requirements, architectures, challenges, standards and solutions." Communications Surveys \& Tutorials, IEEE 13.4 (2011): 584-616. 
[10] Mangold, Stefan, Sunghyun Choi, Peter May, Ole Klein, Guido Hiertz, and Lothar Stibor. "IEEE 802.11 e Wireless LAN for Quality of Service." In Proc. European Wireless, vol. 2, pp. 32-39. 2002.

[11] Elias, Shamsul Jamel, et al. "A Comparative Study of IEEE 802.11 Standards for Non-Safety Applications on Vehicular Ad Hoc Networks: A Congestion Control
Perspective." Proceedings of the World Congress on Engineering and Computer Science. Vol. 2. 2014.

[12] Al-Sultan, Saif, Moath M. Al-Doori, Ali H. Al-Bayatti, and Hussien Zedan. "A comprehensive survey on vehicular Ad Hoc network." Journal of network and computer applications 37 (2014): 380-392. 